\title{
PANAMÁ 2009: ElecCiONES, CRISIS MUNDiAL Y MOVIMIENTOS SOCIALES
}

\author{
Panama 2009: Elections, Global Crisis and Social Movements
}

\author{
MARCO A. GANDÁSEGUI, hijo \\ Centro de Estudios Latinoamericanos (CELA)
}

\begin{abstract}
RESUMEN
En 2009 Panamá se enfrentó a la crisis económica mundial y, a pesar de ella, continuó ejecutando las obras de ampliación del Canal de Panamá que representa una inversión total de US\$ 5.250 millones. La crisis logró abollar el crecimiento económico. Al mismo tiempo los indicadores sociales desmejoraron. A la vez, las políticas públicas continuaron generando más protestas sociales y una tasa de criminalidad ascendente. Se eligió un nuevo Presidente de la República, quien cuestionó con éxito la estructura política tradicional bipartidista. El Presidente Martinelli inició su gestión anunciando la habilitación de 11 bases aeronavales con el fin de "combatir el narcotráfico". La falta de experiencia y cultura política del equipo del Presidente Martinelli pueden ser señales de un debilitamiento de las instituciones democráticas.
\end{abstract}

Palabras clave: Elecciones, crisis económica, Canal de Panamá, bases militares, criminalidad, Presidente Martinelli, democracia.

\begin{abstract}
In 2009 Panama had to deal with the global economic recession and, at the same time, continue widening the Panama Canal at a cost of US\$ 5.25 billion. The Global recesion was able to slow down Panama's economic growth. Meanwhile public policies spurred popular protests and a growing crime rate. Panama elected a new President in 2009 who was able to bring down with success what seemed to be shaping up to be a two-party political structure. On the international front, President Martinelli inaugurated his mandate setting-up 11 air and naval bases to "combat drug trafficking." President Martinelli's administration lack of political culture and expierence can be signs of a weakening of democratic institutions.
\end{abstract}

Key words: Elections, economic recesion, Panama Canal, military bases, crime, President Martinelli, democracy. 


\section{INTRODUCCIÓN}

Las elecciones celebradas en Panamá en el mes de mayo se convirtieron en el centro de atención durante el 2009. El triunfo electoral de Ricardo Martinelli introdujo un nuevo elemento en el ambiente político panameño, ya que desplazó la tradicional pugna entre el Partido Revolucionario Democrático (PRD), fundado por el general Omar Torrijos en 1978, y el Partido Panameñista que fundara el carismático líder Arnulfo Arias en la década de 1930. Su triunfo electoral, sin embargo, se debe en gran parte a la alianza con el Partido Panameñista varios meses antes de las elecciones.

El nuevo presidente introdujo un estilo "empresarial" a su gobierno, poco visto desde los gobiernos de la Coalición Patriótica Nacional de la década de 1950. La derrota del PRD, a su vez, generó una crisis de liderazgo en esa agrupación que sigue siendo el partido político más importante del país.

El nuevo gobierno tuvo que enfrentar de una vez la crisis económica que afecta directamente a EE.UU. y al mundo entero. La tasa de crecimiento del producto interno bruto (PIB) disminuyó del 9 por ciento en 2008 a menos del 3 por ciento en 2009. Sin embargo, los efectos de la crisis no detuvieron los megaproyectos en marcha o que se han anunciado. La ampliación del Canal de Panamá a un costo de US $\$ 5.250$ millones sigue avanzando. Las actividades portuarias siguen su dinamismo, encabezando el movimiento de contenedores a nivel regional. Las inversiones en el sector inmobiliario perdieron el ímpetu extraordinario del lustro pasado pero continuaron creciendo a un ritmo más moderado. Las transacciones del centro bancario siguieron su crecimiento.

La capacidad para amortiguar los efectos de la crisis económica mundial por parte de la economía panameña puede deberse a dos factores principales. Por un lado, las inversiones en la ampliación del Canal que conserva su dinámica gracias a la fuerte relación comercial entre China y EE.UU. A su vez, las exportaciones chinas le imprimen a la Zona Libre de Colón y a los puertos panameños un movimiento muy fuerte. Otro elemento que contribuye al crecimiento económico -con efectos colaterales negativoses el lavado de dinero y el tráfico de ilícitos por territorio panameño. Es una actividad muy difícil de contabilizar pero sus efectos se sienten en la economía informal y en la creciente criminalidad que afecta el país.

De igual manera, EE.UU. ha convertido a Panamá en un centro de actividades militares que complementa sus actividades bélicas en la vecina Colombia. El gobierno panameño anunció la remilitarización de sus fuerzas del orden dando inicio a la construcción de 11 bases aeronavales sobre ambos litorales del país.

La crisis económica mundial tuvo sus efectos sobre el tejido social del país, incrementando el desempleo y la informalidad. Los sectores más golpeados fueron los productivos, tanto manufacturero como agropecuario, que vieron disminuir su contribución al PIB y un incremento en el desempleo. Los efectos de la crisis se sintieron en los sectores de salud, educación y seguridad social, que no lograron presentar propuestas para su inserción en los procesos de cambio. La participación en la captación de riquezas por parte de los trabajadores continuó disminuyendo, creando incertidumbre y un ambiente 
propicio para las protestas. Martinelli anunció que le daría seguimiento a las políticas de subvención social a los sectores de la población que se encuentran por debajo de la línea de pobreza: "La red de oportunidades". Además, anunció un programa de bonos de US $\$ 100$ para toda persona mayor de 70 años de edad sin acceso a servicios sociales: "Cien para 70".

\section{LA SITUACIÓN ECONÓMICA}

Apenas transcurridos los primeros meses de 2009 se sintieron los efectos económicos de la crisis mundial. "La actividad económica de Panamá cayó en un 2,6 por ciento en abril frente al mismo mes del año anterior", según el gobierno. La economía de Panamá sólo creció un 2,5 por ciento en el primer trimestre del año, mientras que cerró el 2008 con un crecimiento del 9,2 por ciento.

La recesión global comenzó golpeando el tráfico comercial del Canal de Panamá. El sector de la construcción, otro de los motores importantes de la economía panameña en los últimos años, acusó una baja del 9 por ciento en 2009. La inversión bajó de casi 2 mil millones en 2008 colocándose en US\$ 1.548 millones en 2009. ${ }^{1}$

El sector más afectado por la recesión fue el comercio marítimo. Los puertos sufrieron una pérdida del 9 por ciento comparado a 2008. Igualmente, la Zona Libre de Colón tuvo un bajón del 9 por ciento. El renglón más afectado por la crisis fue la exportación de frutas "no tradicionales". La baja más significativa fue el rubro melón con el 74 por ciento, seguido por la sandía con un 58,9 por ciento.

El sector turístico acusó un estancamiento que contrastó con el crecimiento espectacular de los años anteriores. La crisis afectó menos la generación de electricidad. En los últimos cinco años (2005-2009) el sector ha crecido a una tasa regular del 6 por ciento.

\section{La reacción del gobierno frente a la crisis económica}

Ante la crisis económica mundial, el gobierno anunció que endeudó al país en 1.110 millones de dólares al suscribir un préstamo con varias agencias internacionales, incluyendo el BID y el Fondo Andino. El préstamo tendría como propósito apoyar a la banca panameña ante los recortes de créditos provenientes de los entes financieros internacionales tradicionales.

El economista y profesor universitario, Juan Jované, planteó la necesidad de ejecutar un plan de emergencia que contemple impulsar la producción en los sectores estratégicos del agro y de la manufactura. Además, planteó la necesidad de invertir en la construcción de planteles de educación e instalaciones de salud. Agregó que el plan de emergencia tiene que estar amarrado a un Plan de Desarrollo Nacional. Las propuestas de Jované no encontraron

1 Una nota interesante sobre la "burbuja" inmobiliaria panameña -en broma y en serio- apareció en la revista norteamericana Time. Tim Rogers, 2010, “Donald Trump Goes on an Adventure in Panama”, Time, 20 de marzo. 
eco en los corredores del gobierno. Jované levantó una propuesta de centroizquierda en el marco de las elecciones de 2009. Fue director general de la Caja del Seguro Social (CSS) en 2003 donde adquirió un perfile nacional por su defensa de los asegurados.

\section{El Canal de Panamá}

La primera década del siglo XXI fue dominada por la incorporación del Canal de Panamá a la economía del país. La vía acuática se enfrentó en 2009 a la peor crisis del comercio marítimo mundial desde la década de 1930. La empresa Lloyd's List informó en enero de 2009 que cinco empresas de transporte marítimo de contenedores redujeron sus rutas debido a la recesión económica que golpea el comercio mundial. Son las navieras APL, Haburg Sud, Hapag-Lloyd, Hyundai Merchant Marine y Evegreen.

Panamá está invirtiendo -en un período de seis años (2007-2013)- un total de US\$ 5.250 millones en la ampliación del Canal de Panamá. La ampliación consiste en la construcción de nuevas entradas, en la realización de dragados y en la habilitación de dos juegos de esclusas con capacidad para recibir barcos de hasta 150 mil toneladas de desplazamiento. En la actualidad, las esclusas del Canal sólo tienen capacidad para recibir barcos con un máximo de 64 mil toneladas. Las esclusas representan el 60 por ciento de la inversión total.

En 2009 existían pronósticos negativos para el Canal. Por ejemplo, la HSBC Global Research, sección de investigaciones del banco sino-británico, hizo proyecciones muy pesimistas, señalando que las tarifas del comercio marítimo sufrirán bajas significativas, poniendo en peligro la existencia de las navieras. HSBC señaló que el comercio marítimo en contenedores se estancaría en 2009 y que en 2010 podría recuperarse un poco. Casi todas las navieras anunciaron pérdidas para 2009.

Cuadro 1: Tránsitos, peajes y toneladas por el Canal de Panamá. 2007-2009

\begin{tabular}{lccc}
\hline & Tránsitos & $\begin{array}{c}\text { Peajes } \\
\text { (millones de US\$) }\end{array}$ & Toneladas (miles) \\
\hline 2009 & 14.342 & $1.438,2$ & 198.014 \\
2008 & 14.702 & $1.317,5$ & 209.763 \\
2007 & 14.721 & $1.183,9$ & 208.220 \\
\hline
\end{tabular}

Fuente: Autoridad del Canal de Panamá.

Las primeras señales de una disminución de los tránsitos por el Canal de Panamá se dieron a mediados de la primera década del siglo XXI. Los tránsitos se estabilizaron en el área de los 14.500 barcos al año. Igualmente, el tonelaje anual que atravesaba la vía acuática se estancó. En 2009 disminuyó en 5 por ciento comparado con el año anterior. Los ingresos por concepto de peajes continuaron aumentando -a una tasa anual de aproximadamente el 10 por ciento- como consecuencia de la política de la Autoridad del Canal de Panamá (ACP) de realizar incrementos periódicos. 
La disminución de las actividades del Canal de Panamá no fue más significativa en gran parte gracias al ritmo de crecimiento de la economía de la R.P. China. El principal socio chino es EE.UU. y parte importante de las importaciones de este país pasan por la vía acuática con destino a la costa oriental norteamericana. Igualmente, el comercio en la otra dirección pasa por el Canal de Panamá. Más de 50 por ciento del flujo de carga que pasa por el Canal va o viene de EE.UU. En segundo lugar, se ubica la carga que va o viene de China con el 15 por ciento del flujo.

Cuadro 2: Flujo de carga de los tres países más importantes que pasa por el Canal de Panamá 2009

\begin{tabular}{lc}
\hline País & Toneladas (miles) \\
\hline EE.UU. & $134.761,8$ \\
China & $46.526,3$ \\
Chile & $25.438,5$ \\
\hline
\end{tabular}

Fuente: Autoridad del Canal de Panamá.

\section{LA SITUACIÓN SOCIAL}

\section{Pauperización y criminalidad}

Según el Centro de Capacitación Social (CCS), "la inseguridad ciudadana se ha convertido en el problema principal que preocupa a la población panameña. Los altos índices de delincuencia ocurridos en el país durante 2009 presentan un panorama desolador". Señala, además, que "la crisis en el sistema de justicia configura un panorama de pocas esperanzas para el respeto del estado de derecho a nivel de la república, donde la concentración de los tres poderes del Estado se fundamentan en un sistema presidencialista y falta de independencia del sistema Judicial y Legislativo".

El CCS agrega que la deserción escolar, la exclusión social de los jóvenes y la falta de participación en la política, la falta de una política de Estado hacia la juventud, sin propuestas de trabajo, han alimentado el crecimiento de las pandillas a nivel nacional. La política del Estado panameño frente a este fenómeno ha sido aumentar el castigo hacia la población juvenil a partir de los doce años, juzgando a los niños y adolescentes como adultos. ${ }^{2}$

"El administrador del Programa de Seguridad Integral (PROSI), Manuel Zamorano, informó que el número de pandillas en Panamá aumentó en los dos últimos años y, además, se ha extendido por todo el territorio nacional. Según esta fuente, en 2007 existían 216 pandillas. En 2009 las autoridades estiman que hay 240 en todo el país, integradas en su mayoría por jóvenes de entre 13 y 21 años.

De acuerdo con Zambrano (sic), las pandillas se han extendido a todas las provincias y, particularmente, hacia Arraiján y La Chorrera, al oeste de la provincia de Panamá.

2 Pérez, Eliecer, 2009. “La pena de prisión en el nuevo Código Penal panameño”, Cuadernos Panameños de Criminología, $\mathrm{N}^{\mathrm{o}} 7$. 
La mayor cantidad de pandillas se ubica en la capital y en San Miguelito. En total operan unas sesenta en estos sectores, de acuerdo con los informes". ${ }^{3}$

\section{Crecimiento económico sin distribución de la riqueza}

Entre 2005 y 2008, la economía panameña creció en un promedio anual del 8 por ciento. El motor detrás de esta expansión se ubicó principalmente en el sector servicios, especialmente los marítimos. El crecimiento económico redujo el desempleo pero no afectó el empleo informal que concentró casi el 50 por ciento de la población económicamente activa. En las áreas rurales y en las urbes del interior del país, el desempleo y el empleo informal superan en un 50 por ciento las cifras de la capital.

En los últimos lustros se ha agudizado la desigualdad en el ingreso de los panameños. Los salarios como parte del producto interno bruto están perdiendo importancia en comparación con las ganancias de los empresarios. En el primer trimestre de 2009 los salarios disminuyeron relativamente y, al mismo tiempo, subió el precio de la canasta básica.

En la actualidad, la prestación de servicios de salud ha colapsado (Ministerio de Salud y CSS), la salud ambiental se encuentra en un espiral descendente (la quiebra de los servicios de basura, el descontrol de enfermedades contagiosas como el dengue y otros problemas) y los indicadores cada día son más alarmantes (han regresado las enfermedades contagiosas, las respiratorias y las tasas de morbilidad han aumentado). El gobierno señala que el presupuesto de salud se incrementa anualmente pero sin obtener los resultados esperados.

\section{Los movimientos sociales}

Los partidos políticos han perdido su capacidad para movilizar a los sectores organizados de la población. En los últimos 20 años se han incrementado los movimientos sociales que expresan sus reivindicaciones en las calles y en los campos. Los trabajadores han rechazado-sin éxito- la imposición de la flexibilización de las relaciones obrero-patronales protestando en las calles de las ciudades del país. ${ }^{4}$ Los gobiernos han respondido con más legislación y represión. Durante la campaña que culminó en mayo de 2009, los candidatos a la Presidencia no se refirieron al problema del costo de la vida, del empleo informal y del empobrecimiento de los trabajadores. Sólo Juan Jované, cuya aspiración a ser incluido en el proceso electoral como candidato independiente fue rechazado por el Tribunal Electoral, denunció la política laboral represiva del gobierno.

En 2009 se multiplicaron las protestas y manifestaciones de múltiples sectores que reivindican acceso a mejores servicios gubernamentales, a sus derechos constitucionales y al desarrollo sostenible. En los centros urbanos hubo un incremento de la criminalidad, producto de la escasez de oportunidades para los jóvenes y de la corrupción que se origina en el tráfico ilegal de drogas, armas y trata de blancas combinados con el control de los juegos de azar.

Tomado de Capacitación Social, http://www.the panama news. Com/pn/v_16/issue_04/opiniones_09. html

4 Quintero, Iván, 2010. Mercado de trabajo, flexibilidad y lucha obrera en Panamá, Panamá: Suntracs. 
La seguridad ciudadana se convirtió en la reivindicación urbana más importante en 2009, desplazando el desempleo y la pobreza. Es seguida de cerca por el transporte público y servicios como el agua potable y la basura. Además, son cotidianas las protestas de educadores y jubilados. Los casos de corrupción se han adueñado de los estamentos encargados de la seguridad haciendo peligrar la estabilidad de ciudades como Panamá y Colón.

El gobierno del Presidente Martinelli ha propuesto un proyecto llamado Metro Bus. El proyecto contempla transportar en una ruta inicial en el área metropolitana de la ciudad de Panamá "15 mil pasajeros en las horas más intensas e incluiría un gran terminal en San Miguelito. Prestaría servicio de 4:30 a.m. hasta las 12:00 (medianoche). Los 21,5 kilómetros y 21 estaciones de la propuesta original quedaron recortadas a 14, para cumplir con un tiempo quinquenal de construcción" ${ }^{5}$

Según Alessandro Ganci, en el área metropolitana "circulan aproximadamente 420.000 vehículos particulares. Se generan aproximadamente 2 millones de viajes diarios, de los cuales la mitad se realiza en transporte público (1.758 autobuses), el 17 por ciento en vehículos particulares y el 20 por ciento a pie".

En las áreas rurales la situación es aún más difícil. Las comunidades campesinas en Azuero y Veraguas han sido desplazadas por proyectos mineros que contaminan sus aguas o proyectos hidráulicos en Chiriquí que inutilizan sus tierras. En 2009 se multiplicaron las protestas de empleados de la salud y de la educación. También se desató un largo conflicto entre el gobierno y los jubilados que culminó con el reconocimiento de una mejor remuneración para éstos.

También se agudizaron los enfrentamientos entre las autoridades y las comunidades que son víctimas de proyectos que no respetan las reglas ambientales del país. En primer lugar se destacan los proyectos mineros que en diferentes puntos del país se desarrollan prácticamente sin supervisión alguna del gobierno. La promoción del turismo también ha colocado a las autoridades en un camino de colisión con las comunidades. ${ }^{6}$

Los conflictos comunitarios tradicionales en torno a reivindicaciones como los servicios de salud, el agua y el transporte también generan conflictos en centros poblados tanto urbanos como rurales. El abandono de sus deberes legales por parte las autoridades encargadas de la distribución de agua potable es objeto de protestas, cortes de vías y manifestaciones públicas casi a diario. Igualmente, la falta de mantenimiento a caminos rurales y calles urbanas genera protestas cotidianas.

Los conflictos laborales también se sintieron en el período. La huelga declarada por los trabajadores de la industria láctea Estrella Azul se extendió debido al intento de la empresa por desconocer los derechos de los obreros. Finalmente se llegó a un acuerdo cuando la patronal negoció un incremento mínimo del salario base. En el sector construcción fueron múltiples los paros de los trabajadores quienes protestaban, entre otros motivos, por las condiciones de inseguridad en que muchos empresarios desarrollan sus actividades. 
Entre marzo y abril, y en medio de la campaña electoral, el gobierno de Martín Torrijos procedió a desalojar a las comunidades del pueblo indígena naso de sus tierras en la provincia de Bocas del Toro.

\section{Pobreza y situación de los indígenas}

La carencia de una política gubernamental coherente contra la pobreza, acompañada de políticas fiscales inadecuadas, han creado situaciones que agravan aún más la ya existente desigual distribución de la riqueza. En 2009 el costo de la canasta básica aumentó en forma significativa. Las medidas tomadas, como la reducción de aranceles y la inversión en estaciones o en una cadena de plantas que conservan en frío los productos agrícolas con destino al mercado, no consiguieron bajar los precios.

Pedro Acosta, presidente de la Unión de Consumidores y Usuarios de la República de Panamá, considera que el costo de la canasta básica en términos generales sigue estando alta, pero que los consumidores no están siendo conscientes de su situación económica y financiera. Por su parte, Vicente Aguirre, presidente del Instituto de Estudios y Defensa del Consumidor, apuntó que los productos suben de precio por el alza en el combustible, pero cuando éste baja, la subida en los alimentos se mantiene.

Panamá tiene una población de doscientos setenta mil indígenas aproximadamente, distribuida en ocho grupos étnicos en todo el país, que aunque poseen diferencias entre sí, también tienen muchos elementos en común. El más evidente de los elementos comunes entre los grupos étnicos es la extrema pobreza, el analfabetismo, la discriminación racial, laboral y social, la desprotección jurídica y el desentendimiento de las autoridades para con sus deberes básicos hacia esta población vulnerable. Los mismos se encuentran marginados de sus derechos constitucionales, civiles, políticos, en fin, son considerados como ciudadanos de segunda clase, producto de la discriminación económica, política y social de los gobernantes de turno. ${ }^{7}$

El desempleo es crónico, los ingresos son escasísimos, la indefensión frente a las catástrofes naturales y la inmisericorde explotación laboral hacen que la expectativa de vida promedio sea más baja en esta población.

Lo anterior va aunado al mínimo respeto por sus costumbres y valores culturales. El sistema educativo persigue la incorporación del indígena a la sociedad y provoca su desaparición paulatina como ente que forma parte de una diversidad cultural. Los pueblos indígenas han resistido la ofensiva de inversionistas durantes varios siglos. Las crisis económicas, la ampliación de las áreas de cultivo y la necesidad de nuevas áreas de comercio presionan a los indígenas. Los indígenas representan una fuente de mano de obra barata y de tierra casi gratuita para terratenientes, ganaderos y recientemente para empresas hidroeléctricas y mineras en sus comarcas.

Ver Clavero, Bartolomé, 2009. “Gobierno panameño y AES-Panamá en el caso Charco La Pava, Quito: Alainet. Endara H., Héctor, 2009. “Lucha indígena y campesina”, Quito: Alainet, 8 de octubre. Valdés, Rodelick, 2009. "Políticas indígenas y el Estado panameño", Quito: Alainet, 22 de octubre. 


\section{Género}

De las 833 mujeres que aspiraron a cargos de elección en 2009, sólo 81 fueron elegidas y la mayoría como representantes de corregimientos (la división político-administrativa más pequeña de la República). Lo anterior, según CCS, evidencia un retroceso desde el punto de vista político para la situación de las mujeres en su acceso al poder. Aunado a ello, la tendencia se confirmó con la existencia de sólo tres mujeres en el gabinete de Ricardo Martinelli.

Cuadro 3: Mujeres postuladas y electas en las elecciones de 2009

\begin{tabular}{lcccr}
\hline \multicolumn{1}{c}{ Cargo de elección } & $\begin{array}{c}\text { Mujeres } \\
\text { postuladas }\end{array}$ & $\begin{array}{c}\text { Candidatos } \\
\text { postulados }\end{array}$ & $\begin{array}{c}\text { Mujeres } \\
\text { electas }\end{array}$ & $\%$ \\
\hline Presidente & 1 & 3 & 0 & 0 \\
Alcalde & 83 & 381 & 7 & 8 \\
Diputado & 53 & 335 & 7 & 13 \\
Representante & 696 & 4.623 & 67 & 10 \\
Total & 833 & 5.340 & 81 & 10 \\
\hline
\end{tabular}

Fuente: Centro de Capacitación Social.

Lo anterior contrasta con el establecimiento de la Ley de Igualdad de Género que fijó en un 30 por ciento la participación femenina en las candidaturas a puestos de elección, realidad que por evidencia no se ha cumplido.

El desempleo se mantiene mayormente entre las mujeres y los niveles salariales de los hombres son mayores a los de las mujeres panameñas. En cuanto a la realidad laboral, según el Observatorio de Género y Economía, de la Universidad de Panamá, el índice de desempleo de las mujeres en 2009 era 8,9 por ciento. Mientras tanto, el desempleo entre los hombres sólo era 5,6 por ciento. Según estadísticas del Observatorio Panameño contra la Violencia de Género (OPVG), adscrito a la Defensoría del Pueblo, las muertes violentas de mujeres van en aumento. En 2008 perdieron la vida 42 mujeres de forma violenta. En 2009 se registró un total de 80 muertes violentas de mujeres (54 de ellas tipificadas como femicidio).

\section{LAS ELECCIONES DE 2009}

En Panamá se celebran elecciones cada cinco años a cuatro niveles en forma simultánea. A nivel nacional hay elecciones para la Presidencia de la República. A nivel de circuitos electorales se eligen 71 diputados para que ocupen sus curules en la Asamblea Nacional. A nivel de distrito se eligen 75 alcaldes. Por último, en cada corregimiento se elige un Representante quien es, a su vez, presidente de su junta comunal y, también, concejal en el Concejo Municipal. 
En el estudio de Pérez y Seligson ${ }^{8}$ se llama la atención sobre el pragmatismo del electorado panameño que pone la democracia al servicio de sus intereses económicos. En el caso de que el régimen político democrático no produzca el bienestar esperado, el electorado panameño puede inclinarse por un gobierno militar. En el estudio de Pérez y Seligson también se destaca el carácter clientelista del electorado panameño. En todas las elecciones la participación supera holgadamente el 70 por ciento de la población con derecho a ejercer el voto. En cambio, en el referéndum convocado para decidir si se aprobaba el proyecto de ampliación del Canal de Panamá sólo llegó a las urnas el 40 por ciento de la población electoral.

Cuadro 4: Resultados de la elección presidencial del 4 de mayo de 2009.

Votos obtenidos para candidatos a Presidente de la República por alianza y partido

\begin{tabular}{lcc}
\hline & Votos & $\%$ \\
\hline Total & 1.586 .427 & 100,0 \\
\hline Ricardo Martinelli & 952.333 & 60,0 \\
Alianza por el Cambio & 94.841 & \\
\hline MOLIRENA & 293.554 & \\
Partido Panameñista & 509.986 & 37,6 \\
CD & 53.952 & \\
Unión Patriótica & 597.227 & \\
\hline Balbina Herrera A. & 553.974 & 2,3 \\
Un País para Todos & 35.459 & \\
\hline PRD & 7.794 & \\
Partido Popular & 36.867 & \\
Partido Liberal & & \\
\hline Guillermo Endara & & \\
Vanguardia Moral de Patria & & \\
\hline
\end{tabular}

Fuente: Tribunal Electoral de Panamá.

\section{Martinelli obtiene una mayoría del 60 por ciento}

Ricardo Martinelli, candidato de la Alianza por el Cambio y del Partido Cambio Democrático, sorprendió al país cosechando el 60 por ciento de los votos emitidos el domingo 3 de mayo de 2009. La candidata del PRD, Balbina Herrera, sólo alcanzó el 37 por ciento. Según el Tribunal Electoral, la participación alcanzó un 73 por ciento de la población con derecho a sufragar.

La mayoría obtenida por Martinelli refleja tres tendencias muy marcadas. Por un lado, el voto castigo contra el PRD. Por el otro, el aporte del Partido Panameñista. Además,

8 Pérez, Orlando y Mitchel Seligson, 2008, Cultura política de la democracia en Panamá, 2008: El impacto de la gobernabilidad, Washington: LAPOP. 
una juventud que votó por una alternativa diferente y que sólo encontró a Martinelli. Fue precisamente en el estrato joven donde Martinelli logró el mayor apoyo a su imagen que proclamaba el "cambio".

Martinelli cuenta, además, con una cómoda mayoría de 42 diputados (de un total de 71) en la Asamblea Nacional. Casi la mitad - un total de 20- son del Partido Panameñista. El PRD -en la oposición- tendrá una tercera parte (26) de los curules de la Asamblea.

Desde la invasión militar norteamericana de 1989, la Asamblea dejó de ser un cuerpo político dinámico. Sus iniciativas dependen casi en su totalidad del Ejecutivo. Martinelli tendrá que demostrar una cualidad que aún se le desconoce: capacidad de dirección política para impulsar su programa de gobierno. ${ }^{9}$

Durante la campaña electoral de 2009 el Presidente Martinelli enfatizó su compromiso con el tratado de libre comercio con EE.UU. Además, prometió "cambiar" el sistema educativo público, los servicios de salud y la seguridad social. En torno al Canal de Panamá no tiene propuesto alterar la política actual. En materia de medio ambiente, cultura, género y ciencia no compartió con el electorado sus preocupaciones. ${ }^{10}$

Su contrincante electoral, Balbina Herrera, también dio garantías de que impulsaría el tratado comercial con EE.UU. Además, se distanció de los gobiernos independientes de la región subrayando su lealtad hacia Washington. La candidata del PRD apoyó los planes para "descentralizar" los servicios públicos que privatizarían la educación, la salud y la seguridad social. Con relación al Canal de Panamá, ambiente y género no presentó novedades.

En la coyuntura electoral, la izquierda política panameña se presentó atomizada, sin poder presentar alternativas viables al pueblo. En 2009, una alianza de fuerzas de izquierda levantó la candidatura de Juan Jované, profesor de Economía de la Universidad de Panamá, quien pretendió presentarse utilizando el mecanismo de la libre postulación. El Tribunal Electoral rechazó la solicitud de Jované señalando que sólo los partidos políticos estaban legalmente autorizados para presentar candidaturas a la Presidencia de la República.

Otro sector de la izquierda, organizado en torno a FRENADESO, rechazó la convocatoria electoral calificándola de un "circo" de las clases dominantes. Hizo un llamado a la abstención o al voto en blanco. Entre las organizaciones pertenecientes a FRENADESO se destaca el poderoso sindicato de los obreros de la construcción, las asociaciones de profesores y maestros, asociaciones estudiantiles y otros sindicatos.

La campaña de Jované contó con el apoyo del Partido del Pueblo (comunistas) y del Partido Alternativa Popular. También recibió el respaldo de poderosos sindicatos de los trabajadores de la industria de las bebidas, con asociaciones de empleados, docentes, grupos estudiantiles y otras organizaciones. ${ }^{11}$

9 Partido Cambio Democrático, 2009, Resumen Ejecutivo: Estrategia para el desarrollo económico y social de Panamá. Propuesta económica 2004-2009, Panamá: Partido Cambio Democrático.

10 Para un tratamiento más a fondo de las elecciones de 2009, ver Leis, Raúl (2009) y Harry Brown A. (2009).

11 Ver Gandásegui, hijo, Marco A. (2009). “Panamá: La izquierda panameña y las elecciones”, ALAINET, 18 de junio. 


\section{Política exterior}

Los resultados de las elecciones panameñas marcaron una tendencia contraria a la que caracterizó al resto de América Latina durante la primera década del siglo XXI. El movimiento del péndulo hacia la izquierda del espectro político incluso fue cuestionado por Martinelli. En política exterior el Presidente Martinelli se pronunció, antes de llegar al Palacio, a favor de definir un eje con varios presidentes conservadores de la región. También se declaró amigo de la política exterior de EE.UU., que serviría de guía para su posición en los foros internacionales. Los aliados más próximos de Panamá, además de EE.UU., serían los gobiernos de Colombia y México. Le seguiría en importancia estratégica Chile, después del triunfo electoral de la derecha en ese país en 2010.

En las Naciones Unidas el gobierno de Martinelli, por primera vez en 40 años, rompió su posición de neutralidad y apoyó las políticas de anexión israelí sobre territorios palestinos. En la Organización de Estados Americanos rompió con el consenso de aislar a Honduras, cuyo gobierno electo fuera depuesto por un golpe militar. EE.UU. logró también que Panamá comenzara a habilitar once bases aeronavales en las costas e islas de ambos océanos que bañan las costas panameñas.

\section{Economía}

Martinelli planteó en su campaña, y después en sus discursos desde la Presidencia, su intención de desarrollar una política que pone los intereses de los empresarios panameños en primer lugar. Presentó una propuesta fiscal destinada a reducir en forma significativa los impuestos al sector empresarial. Además, anunció mayor flexibilización de las relaciones obrero-patronales. Martinelli, sin embargo, no cuenta con un plan de contingencia para enfrentar los efectos de la crisis económica mundial.

Las promesas electorales de Martinelli se enfrentaron a varios obstáculos. El principal son los intereses de los sectores financieros que controlan la mayor parte de la economía. Los empresarios de la banca y de la Zona Libre de Colón, quienes no pagan impuestos, han dado señales de oposición. Desde principios de la década la recaudación tributaria ha crecido gracias al incremento de los peajes del Canal de Panamá. Otra fuente es el impuesto a la renta que incluye el CAIR y el ITBM. Este último constituye la espina dorsal de la propuesta de reforma fiscal con un aumento del 40 por ciento (del 5 al 7 por ciento).

\section{El gabinete de Martinelli}

El Presidente Martinelli tomó posesión el $1^{\circ}$ de julio e inmediatamente puso en práctica una política neoliberal mezclada con un fuerte compromiso con los capitales nacionales. Martinelli y sus colaboradores manifestaron que no tolerarán protestas sociales de los trabajadores, educadores o comunidades. En el marco de las propuestas, los sectores empresariales, que apoyan a Martinelli, se sorprendieron por los nombramientos realizados para ocupar posiciones en su consejo de gabinete. La mayoría de las figuras escogidas no tienen experiencia pública y sus especialidades no cuadran necesariamente con las responsabilidades que van a asumir. 
El consejo de gabinete tiene 13 ministros de Estado (sin incluir los dos nombrados por decreto presidencial). Un total de seis ministros son del Partido Cambio Democrático (CD) y cercanos colaboradores. El vicepresidente del CD, Roberto Henríquez, fue designado ministro de Comercio e Industrias, cartera ligada a las inversiones extranjeras. Martinelli designó a su abogada, Dora Cortés, para encargarse de la cartera de Trabajo.

Los nombramientos que más llamaron la atención fueron los de Salud, donde designó a su médico de cabecera. En Educación nombró a la periodista Lucy Molinar y en Desarrollo Social quedó el popular presentador de televisión, Guillermo Ferrufino. Como ministro de la Presidencia, Martinelli tiene a su jefe de campañas electorales (2004 y 2009), Demetrio Papadimetriu.

\section{Cuadro 5: Gabinete del Presidente Ricardo Martinelli}

Ministerio de Relaciones Exteriores: Juan Carlos Varela (Partido Panameñista)

Viceministro: Meliton Arrocha

Ministerio de Gobierno y Justicia: José Raúl Mulino (Partido Unión Patriótica)

Ministerio de Educación: Lucinda Molinar (Partido Cambio Democrático)

Viceministro: Yolanda de Crespo

Ministerio de Desarrollo Agropecuario: Víctor Manuel Pérez (Partido CD)

Viceministro: Víctor Villarreal

Ministerio de Economía y Finanzas: Alberto Vallarino (Partido Panameñista)

Viceministro: Frank de Lima

Viceministro: Dulcidio de la Guardia

Ministerio de Comercio e Industrias: Roberto Henríquez (Partido Cambio Democrático)

Viceministro: Ricardo Quijano

Viceministro: José Domingo Arias

Ministerio de Salud: Franklin Vergara (Partido Cambio Democrático)

Viceministro: Julio Santamaría

Ministerio de Vivienda: Carlos Duboy (Partido Panameñista)

Viceministro: Jaime Ford Castro

Ministerio de Obras Públicas: Federico José Suárez (Partido Cambio Democrático)

Viceministro: Iván de Icaza

Ministerio de Trabajo: Alma Cortez (Partido Cambio Democrático)

Viceministro: Luis Ernesto Carles

Ministerio de Desarrollo Social: Guillermo Ferrufino (Partido Cambio Democrático)

Viceministra: Susy de Varela

Ministerio de Asuntos del Canal: Rómulo Roux (Partido Cambio Democrático)

Ministerio de la Presidencia: Demetrio Papadimitriu (Partido Cambio Democrático)

Viceministra: María Fábrega

Autoridad de Turismo: Salo Shaman

Autoridad de la Pequeña y Mediana Empresa: Giselle de Calcagno

\section{El nuevo Presidente}

El $1^{\circ}$ de julio el Presidente Ricardo Martinelli al entrar al palacio de Las Garzas (sede de la Presidencia) se encontró con un país inmerso en problemas para promover políticas de 
desarrollo social y económico. Durante varios lustros los gobiernos han enfatizado políticas neoliberales. A esta situación se le agrega, actualmente, la recesión norteamericana que ha paralizado las inversiones e intercambios comerciales a escala mundial.

Los indicadores presentan fuertes deterioros en áreas estratégicas como la educación, la salud, el desarrollo urbano y otros aspectos sociales claves. En materia económica, después de un crecimiento del producto interno bruto promedio del 8 por ciento entre 2005 y 2008, en el presente año se proyecta un incremento de apenas el 2,5 por ciento.

La estrategia neoliberal también agudizó los problemas asociados con el crimen organizado. Un relajamiento de las leyes abrieron las puertas a la corrupción y al abuso en negocios turbios como los juegos al azar, el lavado de dinero y el tráfico de drogas. La violencia, pareja íntima de la corrupción, ha saltado a la palestra multiplicándose los delitos y los crímenes. De la corrupción no se escapan los grandes proyectos públicos, negocios particulares y la vida cotidiana del panameño.

Cuadro 6: Resultados de la elección a diputados del 3 de mayo de 2009.

Distribución de curules legislativas por partido político

\begin{tabular}{|c|c|c|c|c|}
\hline \multirow{2}{*}{$\begin{array}{c}\text { Alianza, partido político y libre } \\
\text { postulación }\end{array}$} & \multicolumn{2}{|c|}{ Total de votos válidos } & \multicolumn{2}{|c|}{ Total de curules } \\
\hline & Cantidad & $\%$ & Cantidad & $\%$ \\
\hline Totales & 1.504 .355 & 100,0 & 71 & 100,0 \\
\hline Un País para Todos & 611.135 & 40,6 & 27 & 38,0 \\
\hline PRD & 537.426 & 35,7 & 26 & 36,6 \\
\hline Popular & 55.598 & 3,7 & 1 & 1,4 \\
\hline Liberal & 18.111 & 1,2 & 0 & 0,0 \\
\hline Alianza por el Cambio & 842.667 & 56,0 & 42 & 59,2 \\
\hline Cambio Democratico & 352.319 & 23,4 & 14 & 19,7 \\
\hline Unión Patriótica & 85.609 & 5,7 & 4 & 5,6 \\
\hline Molirena & 70.457 & 4,7 & 2 & 2,8 \\
\hline Panameñista & 334.282 & 22,2 & 22 & 31,0 \\
\hline Vanguardia Moral de la Patria & 14.760 & 1,0 & 0 & 0,0 \\
\hline Libre postulación & 35.793 & 2,4 & 2 & 2,8 \\
\hline
\end{tabular}

Fuente: Tribunal Electoral de Panamá.

En materia política Martinelli pretende no sólo ser el gran ganador de la contienda electoral del pasado 3 de mayo de 2009. Ha enviado mensajes en el sentido de querer desplazar al Partido Revolucionario Democrático (PRD) -derrotado en las elecciones de mayo- del centro de gravedad de la vida política panameña. 
Durante los primeros meses de su gestión dejó la Asamblea en manos del Partido Panameñista. En el caso de la Corte Suprema de Justicia tuvo oportunidad de nombrar dos magistrados para establecer una mayoría a su favor entre los nueve miembros de ese poder del Estado. Las elecciones de mayo de 2009 le dieron un cómodo margen sobre la oposición en la Asamblea Nacional de Diputados.

El Presidente Martinelli hizo su campaña electoral pregonando el "cambio". Si fracasa le dará paso expedito a una nueva generación para asumir los desafíos. Más aún, surgirá una generación de panameños de una clase diferente de hombres y mujeres llamada a enrumbar el país en otra dirección que beneficie a todos. La extrema derecha está condenada a pasar a la historia como un desliz producto de las malas políticas neoliberales aplicadas en los últimos 20 años.

La campaña electoral tuvo un alto costo para los principales contendientes. Según la Contraloría General de la República, “las fuerzas políticas de Panamá gastaron en el primer trimestre de 2009 un total de 110,1 millones de dólares en propaganda para los comicios del 3 de mayo". Del total, "las televisoras fueron las más beneficiadas con ingresos por 62,4 millones de dólares, seguidas de los diarios con 19,9 millones, mientras que las radiodifusoras y revistas captaron el resto".

\section{EL SIGNIFICADO DE LAS ELECCIONES DE 2009 PARA LA DEMOCRACIA}

\section{Martinelli y el giro a la derecha en Panamá}

Muchos se preguntan si Ricardo Martinelli representa un giro hacia la derecha con relación al gobierno saliente. La misma pregunta se la hacen planteando si el triunfo de Martinelli representó el triunfo de una corriente de derecha que deja atrás la propuesta de más neoliberalismo de Balbina Herrera, abanderada del PRD. El círculo que rodea a Martinelli está compuesto por empresarios que se declaran ideológicamente de derecha. El equipo en apariencia no cuenta con tecnócratas salidos de las escuelas "neoliberales", lo que no quiere decir que los despachos del sector económico del gobierno no se encuentren bien equipados con especialistas del "Consenso de Washington".

La victoria de Martinelli redefinió la derecha en Panamá. Desde 1994, con el triunfo de Pérez Balladares, se perfilaron dos tendencias en la derecha política. Por un lado, los neoliberales que encontraron su nicho de poder en las filas del PRD. Por el otro, una derecha más tradicional vinculada al mercado nacional, ligada al Partido Panameñista. Desde la invasión norteamericana (1989) cada uno gobernó en forma alternada dos períodos de cinco años. Sus gobiernos no se destacaron mucho por sus logros. Más bien queda el recuerdo del desgreño administrativo y la corrupción.

La nueva administración de Martinelli se encuadra ideológicamente con la llamada derecha tradicional. Creó el Partido Cambio Democrático en 1998 y en 2004 lanzó su propia candidatura y fue derrotado en forma contundente. 
El triunfo electoral de Martinelli en 2009 puede deberse, en gran parte, a la crisis interna del Partido Panameñista que se agudizó como consecuencia de los cambios estructurales generados por las políticas neoliberales (énfasis en la economía de servicios, desaparición del mercado interno).

\section{FRENADESO explora futuro electoral}

En la izquierda, Saúl Méndez, dirigente obrero de la construcción, señala que "FRENADESO explorará, de manera amplia, abierta, sin precondiciones de ningún tipo, la posibilidad de construir, junto a otras fuerzas populares, gremiales y personalidades democráticas y progresistas, un instrumento político para irrumpir con fuerza en el torneo electoral y disputarle el control que sobre el Gobierno han ejercido hasta el presente los partidos corruptos de la burguesía, caracterizados por el robo y su desprecio a los intereses de las masas populares". De esta manera, Méndez, quien es también coordinador de FRENADESO, anunció la intención que tiene el frente popular de buscar una alternativa electoral.

Los sectores populares no han tenido éxito en las contiendas electorales panameñas. Aun cuando han logrado grandes triunfos mediante la movilización popular, su experiencia en el terreno electoral ha sido infructuosa.

Méndez aclaró "que no se trata de convertir a FRENADESO en partido político". La estrategia que anunciara el dirigente del Sindicato de los Trabajadores de Construcción (SUNTRACS) consiste en "dialogar, reflexionar y debatir con otras fuerzas populares, democráticas y progresistas (sobre) la conveniencia de construir un instrumento político sin dueños". FRENADESO nació hace seis años con la crisis de la Caja de Seguro Social (CSS) que generó protestas masivas de la población y, especialmente, por parte de los trabajadores del país.

Méndez fue el orador de fondo en el II Congreso Nacional de FRENADESO a mediados de agosto de 2009. Enfatizó que quieren "darle salida real al descontento popular y a las ansias y voluntad de cambio del pueblo e impedir que éste siga canalizado por los embaucadores y los falsos profetas de los partidos tradicionales". Según Méndez los oligarcas panameños "trafican con las luchas populares y engañan al pueblo en base a una calculada campaña mediática de los principales centros de desinformación del país y de una multimillonaria campaña publicitaria".

Otro dirigente de FRENADESO, el profesor Andrés Rodríguez, presidente de la Asociación de Profesores, señaló que "hay condiciones para iniciar una organización política basada en la "unidad de los sectores campesinos, indígenas y ambientalistas que se oponen a los proyectos depredadores". Rodríguez denunció la "partidocracia" e, igualmente, "a los partidos corruptos de la clase dominante". Señaló la necesidad de "construir un instrumento político electoral como alternativa".

El Partido Alternativa Popular (PAP) saludó de manera positiva el pronunciamiento de FRENADESO y se ofreció a dialogar en torno a un esfuerzo conjunto para construir el "instrumento político" anunciado por el frente popular. Según Olmedo Beluche, 
dirigente del PAP, "un acuerdo es posible y necesario" entre todos los sectores del movimiento popular.

\section{Los primeros meses del Presidente Martinelli}

Durante su campaña presidencial adoptó como lema la palabra "cambio" que fue acogida por el electorado de manera muy positiva. Los panameños están agotados por la violencia, corrupción y desorden producto de las políticas públicas de los gobiernos que lo antecedieron. Durante los primeros seis meses como gobernante, sin embargo, Martinelli apretó el acelerador sin disminuir la violencia, la corrupción y el desorden a nivel de la cosa pública.

Las cifras que arrojan las estadísticas sobre violencia siguen aumentando. La política pública de control del tráfico de ilícitos, de las pandillas y del juego de azar no se han alterado. Al contrario, el Ministerio de Gobierno y Justicia anunció una inversión de 100 millones de dólares para construir nuevas instalaciones penitenciarias donde detendrá la creciente población delictiva. Se siguen aplicando las mismas políticas ("mano dura", "calles seguras" y otras) que no tienen relación alguna con la violencia y el crimen organizado.

Martinelli prometió poner fin a la corrupción y lo que ha hecho es seguir trabajando en la misma dirección. Antes de cumplir los 60 días en Palacio, Martinelli aprobó gastos por 50 millones de dólares mediante "compras directas" sin pasar por las licitaciones que exige la ley. En el plano del nepotismo, nombró a familiares suyos y de sus colaboradores más allegados en embajadas y consulados, siguiendo las tradiciones de los gobernantes panameños.

\section{Democracia y economía}

Las promesas del Presidente Martinelli en torno a la solución de los problemas básicos como el empleo informal y la crisis de la familia panameña siguen a la espera. En el Ministerio de Trabajo no hay preocupación alguna por enfrentar la situación creada por las políticas de los gobiernos anteriores. Martinelli insinuó ante los empresarios que se flexibilizará aún más la relación obrero-patronal. El equipo económico de Martinelli no reconoce la crisis económica que afecta al mundo y ha insistido que la estructura financiera de Panamá saldrá sin ser afectada.

En materia de política social, suspendió hasta principios de 2010 los programas del gobierno anterior relacionados con la "Red de oportunidades" que repartía subsidios a las poblaciones más empobrecidas. En los sectores de Educación y Salud, así como Seguridad Social, impulsa programas que le permitan reducir costos, mediante reformas curriculares y competitividad entre los médicos. Incluso, coqueteó con la fusión de las entidades encargadas de Cultura y Turismo, señalando que la cultura es una mercancía que pueden comprar los turistas que llegan a nuestras costas.

Martinelli llegó a la Presidencia sin sentar las bases de una política que le permita cumplir con sus promesas de campaña. Gobierna sin máscaras o coberturas populistas 
(panameñistas) o social demócratas (PRD). Su estilo de gobierno es pragmático. Si los sectores mayoritarios quieren dádivas él buscará la manera de distribuirlas. Al mismo tiempo, sin embargo, rechaza el diálogo y la participación de otros sectores organizados de la sociedad. Pareciera que sigue los patrones descubiertos por las encuestas de Pérez y Seligson que privilegian un estilo de gobierno que no considera como ingrediente esencial la democracia.

La clase empresarial se ha tomado el poder y es probable que actúe según sus intereses durante los próximos años. Martinelli ha dicho que no extenderá puentes de entendimiento con otros sectores. Esta táctica política puede traer problemas. Martinelli aún tiene la alternativa de buscar el diálogo con los diferentes sectores de la sociedad que incluya las asociaciones civiles, trabajadores, indígenas y otros.

Panamá, 30 de abril de 2010.

\section{REFERENCIAS}

Beluche, Olmedo, 2009. "Reseña histórica de las reformas neoliberales a la legislación laboral panameña (1971-2004)", Tareas, No 133, septiembre-diciembre (Panamá).

Bosco Bernal, Juan, 2009. "La universidad latinoamericana: Situación, tendencias y perspectivas", Tareas, $N^{\circ} 131$, enero-abril (Panamá).

Castillo Galástica, Adán, 2009. “Información, conocimiento y manipulación: Plataforma para el estudio mediático en tiempos electoreros", Revista Cultural Lotería, $\mathrm{N}^{\circ} 482$, enero-febrero (Panamá).

Castillo B., Damián, 2009. "Oralidad, historia y cultura Bribri”, Revista Cultural Lotería, No 482, enerofebrero (Panamá).

Castillo F., Dídimo, 2009. Los nuevos trabajadores precarios, Toluca: Universidad Autónoma del Estado de México.

Castillo C., Janio, 2009. Ruta de tránsito y la formación social panameña, 1980-1999, Guatemala: FLACSO.

Centro de Capacitación Social, 2010. Situación de los derechos humanos en Panamá en 2009, Panamá: CCS (18 de marzo).

Clavero, Bartolomé, 2009. “Gobierno panameño y AES-Panamá en el caso Charco La Pava, Alainet, Quito: Alainet. 29 de julio.

Comité de Familiares de Desaparecidos de Panamá Héctor Gallego (COFADEPA-HG), 2009. “Breve análisis de las causas del golpe militar en Panamá", Tareas, Nº 131, enero-abril (Panamá).

Maud de León, Elda, 2009. "Educación: Diez mitos y una propuesta", Revista Cultural Lotería, $\mathrm{N}^{\circ} 482$, enero-febrero (Panamá).

Endara H., Héctor, 2009. "Lucha indígena y campesina", Alainet, Quito: Alainet, 8 de octubre.

Figueroa Navarro, Alfredo, 2009. "Los estudios históricos sobre el siglo diecinueve panameño", Tareas, $\mathrm{N}^{\mathrm{0}} 132$, mayo-agosto (Panamá).

Gandásegui, hijo, Marco A. 2009. "Panamá: La izquierda panameña y las elecciones", Alainet, 18 de junio.

Gandásegui, hijo, Marco A. 2009. “Imperialismo norteamericano y la invasión a Panamá de 1989”, Tareas, $\mathrm{N}^{\mathrm{o}} 133$, agosto-diciembre (Panamá).

Gandásegui, hijo, Marco A. 2009. "El militarismo en Panamá", Tareas, № 131, enero-abril (Panamá).

Herrera J., Ligia, 2009. "Colón: La provincia olvidada", Tareas, Nº 132, mayo-agosto (Panamá).

Herrera, Luis C., 2009. Políticas de control en la democracia panameña (1994-2004), Panamá: IDEN (Universidad de Panamá).

Leis, Raúl, 2009. "Panamá: Caja negra electoral”, Nueva Sociedad, No 222, julio-agosto.

Luna, Clara Inés y Salvador Sánchez, 2009. "Panamá: paraíso imperfecto", Revista de Ciencia Política (Santiago de Chile), Vol. 29, No 2, pp. 533-564. 
Manduley, Julio, 2009. Panamá, estructura, coyuntura, conflicto, clases y política, Panamá: Centro de Estudios Estratégicos.

Menéndez Franco, Álvaro, 2009. “Sustentación del grito de Rufina Alfaro y la Independencia de Panamá de España", Revista Cultural Lotería, № 482, enero-febrero (Panamá).

Ministerio de Desarrollo Social (MIDES), 2009. La arquitectura del cambio institucional del MIDES, 20042009, Panamá: MIDES.

Miró G., Carmen A., 2009. “El adulto mayor en Panamá: evolución reciente y perspectivas al 2030-2035", Tareas, $\mathrm{N}^{\circ} 132$, mayo-agosto (Panamá).

Miró G., Carmen A., 2009. Antología. América Latina, población y desarrollo, Bogotá: CLACSO.

Partido Cambio Democrático, 2009. Resumen Ejecutivo: Estrategia para el desarrollo económico y social de Panamá. Propuesta económica 2004-2009, Panamá: Partido Cambio Democrático.

Pérez, Eliecer, 2009. "La pena de prisión en el nuevo Código Penal panameño", Cuadernos Panameños de Criminología, $\mathrm{N}^{\mathrm{o}} 7$.

Pérez, Orlando y Mitchel Seligson, 2008. Cultura política de la democracia en Panamá, 2008: El impacto de la gobernabilidad, Washington: LAPOP.

Quintero, Iván, 2010. Mercado de trabajo, flexibilidad y lucha obrera en Panamá, Panamá: Suntracs.

Turner, Anayansi, 2009. "El derecho de huelga en el Canal de Panamá", Tareas, No 133, septiembrediciembre (Panamá).

Valdés, Rodelick, 2009. "Políticas indígenas y el Estado panameño", Alainet, Quito: Alainet, 22 de octubre.

Yao, Julio, 2009. "Para entender la invasión de EE.UU. a Panamá", Tareas, No 133, agosto-diciembre (Panamá).

Marco A. Gandásegui, hijo. Profesor de Sociología en la Universidad de Panamá e investigador asociado del Centro de Estudios Latinoamericanos (CELA) "Justo Arosemena". 
